\title{
A COUNTER EXAMPLE TO A CONJECTURE OF JOHNS
}

\author{
CARL FAITH * AND PERE MENAL ${ }^{\dagger}$ \\ (Communicated by Maurice Auslander) \\ Dedicated to Carmen Brufal
}

\begin{abstract}
In this paper, we construct a counter example to a conjecture of Johns to the effect that a right Noetherian ring in which every right ideal is an annihilator is right Artinian. Our example requires the existence of a right Noetherian domain $A$ (not a field) with a unique simple right module $W$ such that $W_{A}$ is injective and $A$ embeds in the endomorphism ring $\operatorname{End}\left(W_{A}\right)$. Then the counter example is the trivial extension $R=A \ltimes W$ of $A$ and $W$. The ring $A$ exists by a theorem of Resco using a theorem of Cohn. Specifically, if $D$ is any countable existentially closed field with center $k$, then the right and left principal ideal domain defined by $A=D \otimes_{k} k(x)$, where $k(x)$ is the field of rational functions, has the desired properties, with $W_{A} \approx D_{A}$.
\end{abstract}

\section{INTRODUCTION}

It is well known that over a commutative ring, every Noetherian module with essential socle is Artinian. Although this is not true for arbitrary right Noetherian rings, some positive results have been obtained by Ginn and Moss [6] when the ring is right and left Noetherian. Johns [7, Theorem 1], by using a result of Kurshan [8, Theorem 3.3], showed that a right Noetherian ring is right Artinian, provided that every right ideal is a right annihilator. Ginn [5] had showed that Kurshan's result was false; however, Ginn's example does not provide a counter example to John's theorem. Therefore, the validity of John's theorem was already doubtful. The main result of this paper is to give an explicit counter example to that theorem.

For simplicity, a right Noetherian ring $R$ will be called a right Johns ring if every right ideal of $R$ is the right annihilator of a subset of $R$.

Received by the editors June 12, 1990 and, in revised form, March 29, 1991.

1991 Mathematics Subject Classification. Primary 16A33, 16A34; Secondary 16A39, 16A52.

The work of the first author was supported by three grants: from the Spanish Ministry of Science and Education, Centre Rerca Matematica, and a Rutgers Faculty Academic Semi Sabbatical (FASP).

The work of the second author was partially supported by grant CICYT PB 86-0353-C02-01.

This paper was written during the authors' attendance at the Algebra Semester at the Centre de Recerca Matemàtica in Fall 1989.

${ }^{*}$ I received the final revisions from Pere the week before his tragic death in an automobile accident. The dedication, to his mother, was made when the manuscript was submitted. See the Note preceding the references.

${ }^{\dagger}$ Deceased April 4, 1991. 
Section 2 of this paper gives some positive results. For example, we show that under some additional hypotheses a right Johns ring must be right Artinian (Proposition 3.3). This is used to prove in Theorem 3.4 that Theorem 2 of Johns [7] is correct, even though his proof depends on the false Theorem 1.

Throughout this paper, $R$ denotes a ring with 1 . For any subset $X$ of $R$, we let

$$
r_{R}(X)=\{r \in R: x r=0 \text { for all } x \in X\}
$$

be the right annihilator of $X$ in $R$; the left annihilator $l_{R}(X)$ is defined similarly. We also define $r_{M}(X)$ and $l_{M}(X)$, in the obvious way, when $X$ is a subset of $R$ and $M$ is a left or right $R$-module, respectively.

For any right $R$-module $M$, we write $M_{R}$ to indicate that $M$ is a right $R$-module, and we write $\operatorname{Soc}(M)$ for its socle. The Jacobson radical of a ring $R$ is denote by $J(R)$, or simply $J$, when it is clear from the context.

Recall that a ring $R$ is a right $V$-ring provided that every simple right $R$ module is injective.

\section{THE COUNTER EXAMPLE}

Because our counter example is a trivial extension of a domain, our first aim is to characterize those trivial extensions of domains that are right Johns. We remark, however, that the reader who is strictly interested in the counter example needs to consider the "if" part of the following result only.

Theorem 2.1. Let $A$ be a right Noetherian domain with an A-bimodule $W$ such that $W_{A}$ is simple. Then $A \ltimes W$, the trivial extension of $W$ by $A$, is right Johns if and only if $W_{A}$ is the unique simple right A-module and $A$ is a right $V$-domain.

In the proof of Theorem 2.1, we use the following

Lemma 2.2. (i) [7] If $R$ is a right Johns ring, then $\operatorname{Soc}(R)$ is an essential right ideal of $R$.

Moreover, $J(R)$ is a nilpotent ideal such that $r_{R}(J)=l_{R}(J)=\operatorname{Soc}(R)$.

(ii) $l_{R}(\operatorname{Soc}(R))=r_{R}(\operatorname{Soc}(R))=J$.

Proof. (i) That $J$ is nilpotent is Lemma 1 of [7]; that $l_{R}(J)$ is an essential right ideal is John's Lemma 2; and that $r_{R}(J)=l_{R}(J)=\operatorname{Soc}(R)$ is John's Lemmas $3,4$.

(ii) Since every maximal right ideal is a right annihilator, we see that every simple right $R$-module embeds in $R$. Hence, $r_{R}(\operatorname{Soc}(R))=J$. By (i), $J \subseteq l_{R}(\operatorname{Soc}(R))$. If $x \in l_{R}(\operatorname{Soc}(R))$, then $\operatorname{Soc}(R) \subseteq r_{R}(x)$. Since $\operatorname{Soc}(R)$ is essential, so is $r_{R}(x) . R$ being right Noetherian, this implies $x \in J$. Therefore, $l_{R}(\operatorname{Soc}(R))=J$.

Proof of Theorem 2.1. The "if" part. Since $A$ is right Noetherian and $(0, W)$ is a simple right $R$-module, $R$ is right Noetherian. Since $A$ is a right $V$-ring having a unique simple right $A$-module, we see that $A$ is a simple ring, so $l_{A}(W)=0$. Then, because $W$ is a simple right $A$-module, $a W=W$ for all $a \in A \backslash\{0\}$. Hence, $J=(0, W)$ is an essential simple right ideal of $R$.

Let $I$ be a nonzero right ideal of $R$. Since $J=(0, W)$ is a simple right $R$-module, either $I=J$ or $I \supset J$. In the former, we see that $I$ is a right annihilator. In the second case, since $A$ is a domain, $l_{R}(I) \subseteq J=(0, W)$. 
Thus $l_{R}(I)=l_{(0, W)}(I / J)$. Since $(0, W)$ is a cogenerator of $\operatorname{Mod} A, I / J=$ $r_{A}\left(l_{(0, W)}(I / J)\right)$ (see [4, Proposition 23.13]). If $x \in r_{R}\left(l_{R}(I)\right)$, then $x+J \in I / J$; that is, $x \in I$. Thus $R$ is right Johns.

The "only if" part. Write $R=A \ltimes W$. Note that $(0, W)$ is a simple right $R$-module. Since any simple right $R$-module embeds in $R$, we see that $W$ is "the unique" simple right $A$-module. The fact that $A$ is a right $V$-ring follows from the following general result.

Theorem 2.3. If $R$ is an arbitrary right Johns ring, then $R / J$ is a right $V$-ring.

By Lemma 2.2(i), $\operatorname{Soc}(R)$ is an essential finitely generated right ideal of $R$. Since $\operatorname{Soc}(R)$ is an ideal of $R$, it is an $R$-bimodule, so Lemma 2.2(ii) implies that $\operatorname{Soc}(R)$ is an $R / J$-bimodule. Since $R$ is right Johns, every simple right $R$-module embeds in $\operatorname{Soc}(R)$. Thus, the result will follow by proving that $\operatorname{Soc}(R)$ is injective as a right $R / J$-module. Write $E$ for the injective hull of $\operatorname{Soc}(R)$ (as right $R / J$-modules). Assume $x \in E \backslash \operatorname{Soc}(R)$. Since $E$ is a right $R$-module, $r_{R}(x)$ is a right ideal of $R$ containing $J$. Hence Lemma 2.2(i) implies $l_{R}\left(r_{R}(x)\right)=l_{\operatorname{Soc}(R)}\left(r_{R}(x)\right)$. By using the fact that $R$ is right Johns, we have that $r_{R}(x)=r_{R}\left(l_{\operatorname{Soc}(R)}\left(r_{R}(x)\right)\right)$. This implies that the right $R / J$-module $M=x(R / J) \approx R / r_{R}(x)$ embeds into a direct product of copies of $\operatorname{Soc}(R)$. Since $M \subseteq E, \operatorname{Soc}(M)$ is an essential Artinian submodule of $M$. Therefore, $M$ can be embedded in a finite product of copies of $\operatorname{Soc}(R)$. Hence, $M$ is semisimple Artinian, but then $M=\operatorname{Soc}(M) \subseteq \operatorname{Soc}(E)=\operatorname{Soc}(R)$. Thus $x \in \operatorname{Soc}(R)$, which is not the case.

We finally come to the crux of the construction. We will use Resco's theorem [10] and a theorem of Cohn on existentially closed fields (see [2]).

Corollary 2.3. Let $D$ be any field over a central field $k$ and $A=D \otimes_{k} k(x)$, where $k(x)$ is the field of rational functions.

(i) If $a \in D$ is transcendental over $k$, then $D$ is a simple right A-module whose endomorphism ring is isomorphic to $C_{D}(a)$, the centralizer of a in $D$.

(ii) If $D$ is a countable existentially closed field with center $k$, then $D$ is an A-bimodule that is simple as a right A-module. Further, $A \ltimes D$ is a non-Artinian right Johns ring.

Proof. (i) Let $a \in D$ transcendental over $k$. Then $D$ is a right $A$-module for which

$$
d\left(d^{\prime} \otimes p(x)\right)=p(a) d d^{\prime}
$$

for all $d, d^{\prime} \in D$ and $p(x) \in k(x)$. Clearly $D$ is simple. Now an element of $C_{D}(a)$ gives (by left multiplication on $D$ ) an $A$-endomorphism of $D$. Hence $C_{D}(a) \subseteq \operatorname{End}\left(D_{A}\right)$. Conversely, let $\psi$ be an $A$-endomorphism of $D$. Clearly $\psi$ is left multiplication by an element $d \in D$, but then

$$
d a-a d=\psi(a)-a \psi(1)=\psi(1(1 \otimes x))-\psi(1)(1 \otimes x)=0 ;
$$

that is, $d \in C_{D}(a)$.

(ii) Since $D$ is a countable existentially closed field over $k$, it follows from [2, Theorem 6.2.7] and the remark after that there is a $k$-algebra embedding 
$\alpha: D \hookrightarrow C_{D}(a)$. We also have a $k$-algebra embedding $\beta: k(x) \hookrightarrow C_{D}(a)$ in which $x$ maps to $a$. Now, $A$ being simple, we obtain a $k$-algebra embedding

$$
\gamma=\alpha \otimes \beta: A \hookrightarrow C_{D}(a) \approx \operatorname{End}\left(D_{A}\right),
$$

which converts $D$ into an $A$-bimodule, as required.

By [10], $A$ is a non-Artinian $V$-domain with a unique simple right $A$ module. The result now follows from the above paragraph and Theorem 2.1.

Regarding (ii), we remark that the $V$-domains of Cozzens [3] and Osofsky [9] do not suffice for Corollary 2.3(ii) because their simple modules have commutative endomorphism rings.

\section{SOME POSITIVE RESULTS}

Proposition 3.3 gives a necessary and sufficient condition for a right Johns ring to be right Artinian and also states that a right Johns ring with finite left Goldie dimension must be right Artinian. The latter is the key point in our proof of Theorem 2 in [7]. John's proof in [7] is not correct since it uses that a right Johns ring is right Artinian. However, this theorem is true.

We require some previous results.

By a minimal left annihilator we mean a minimal member in the set of all nonzero left annihilators of subsets of $R$. It is clear that the right annihilator of a minimal left annihilator is actually maximal in the set of all proper right annihilators of $R$; that is, a maximal right annihilator.

Proposition 3.1. Let $W=\bigoplus_{i=1}^{n} W_{i} \subseteq R$ be a maximal direct sum of minimal left annihilators $W_{i}$. Then $\bigcap_{i=1}^{n} r_{R}\left(W_{i}\right)$ is the intersection of all maximal right annihilators of $R$.

Proof. Let $I$ be any maximal right annihilator. Then $l(I)$ is a minimal left annihilator. Hence $l(I) \cap W \neq 0$. Let $0 \neq v \in l(I) \cap W$, and write

$$
v=w_{1}+\cdots+w_{n} \quad\left(w_{i} \in W_{i}\right) .
$$

Since $I$ is a maximal right annihilator and $I \subseteq r_{R}(v), I=r_{R}(v)$. Similarly, if $w_{i} \neq 0$, then $r_{R}\left(w_{i}\right)=r_{R}\left(W_{i}\right)$ is a maximal right annihilator and clearly

$$
I=r_{R}(v) \supseteq \bigcap_{i=1}^{n} r\left(W_{i}\right),
$$

i.e., every maximal right annihilator contains $\bigcap_{i=1}^{n} r\left(W_{i}\right)$. This proves that $\bigcap_{i=1}^{n} r\left(W_{i}\right)$ is the intersection of all maximal right annihilators.

Corollary 3.2. Let $R$ be a ring that contains a copy of each simple right $R$ module. If $R$ is of finite left Goldie dimension, then $R$ is semilocal (i.e., $R / J$ is semisimple Artinian).

Proof. Assume $R$ contains a copy of each simple right $R$-module. Then every maximal right ideal is a right annihilator so every maximal right annihilator is actually maximal. Since $R$ is of finite left Goldie dimension, it follows from Proposition 3.1 that $J$ can be written as a finite intersection of maximal right ideals; that is, $R$ is semilocal. 
Proposition 3.3. (i) Let $R$ be a right Johns ring. Then $R$ is right Artinian if and only if $r_{R}(a)=0$ implies $a R=R$ for every $a \in R$.

(ii) If $R$ is right Johns with finite left Goldie dimension, then $R$ is right Artinian.

(iii) A semilocal right Johns ring is right Artinian. ${ }^{1}$

Proof. Write $\bar{R}=R / J(R)$.

(i) Suppose $R$ is right Johns. The "only if" part follows from the well-known fact that every element of a right Artinian ring whose right annihilator is 0 is invertible.

Conversely, let $a \in R$ be with $r_{\bar{R}}(\bar{a})=0$. Then, since $\bar{R}$ is a semiprime right Noetherian ring, $l_{\bar{R}}(\bar{a})=0$. Therefore,

$$
J=\{x \in R: x a \in J\} .
$$

By Lemma $2.22(\mathrm{ii}), J=l_{R}(\operatorname{Soc}(R))$, so $J=l_{R}(a \operatorname{Soc}(R))$. Now we use the fact that every right ideal is a right annihilator to deduce that $\operatorname{Soc}(R)=a \operatorname{Soc}(R)$. But $\operatorname{Soc}(R)$ is finitely generated, so $r_{R}(a) \cap \operatorname{Soc}(R)=0$. By Lemma 2.2(i), $\operatorname{Soc}(R)$ is an essential right ideal, so $r_{R}(a)=0$. By hypothesis, $a$ is invertible in $R$. Thus $R / J$ is its own classical ring of quotients. Hence $R / J$ is semisimple Artinian. By Lemma 2.2(i), $J$ is nilpotent. Since $R$ is right Noetherian, we see that $R$ is right Artinian. This also suffices for (iii).

(ii) It is immediate from Corollary 3.2 and the latter arguments above.

Theorem 3.4. Let $R$ be a ring satisfying the maximum condition on right annihilators, and

(i) every principal right ideal is a right annihilator.

(ii) $r_{R}\left(I_{1} \cap I_{2}\right)=r_{R}\left(I_{1}\right)+r_{R}\left(I_{2}\right)$ for each pair of left ideals $I_{1}, I_{2}$ of $R$.

Then $R$ is quasi-Frobenius

Proof. If $R$ is right Artinian, then the result derives from [1, Theorem 4.1]. Thus it suffices to show that $R$ is right Artinian. By [7, Lemma 5], $R$ is right Noetherian in which right ideal is a right annihilator. Hence $R$ is right Johns. By Proposition 3.3(ii), we have only to show that $R$ is of finite left Goldie dimension. For if $\bigoplus_{i=1}^{\infty} R a_{i} \subseteq R$, we set

$$
I_{n}=r\left(a_{n}, a_{n+1}, \ldots\right) \text { for all } n \geq 1 \text {. }
$$

Since $R$ is right noetherian, there exists $m \geq 1$ such that $I_{k}=I_{m}$ for all $k \geq m$. Hence

$$
r\left(a_{k+1}, \ldots\right) \subseteq r\left(a_{k}\right)
$$

for all $k \geq m$. Since

$$
\left(\bigoplus_{i \geq k+1}^{\infty} R a_{i}\right) \cap R a_{k}=0
$$

(ii) implies

$$
r\left(a_{k+1}, \ldots\right)+r\left(a_{k}\right)=R .
$$

Then (1) yields $r\left(a_{k}\right)=R$; that is, $a_{k}=0$ for all $k \geq m$. This proves that the left Goldie dimension of $R$ is finite, as required.

\footnotetext{
${ }^{1}$ This is immediate from the work of Johns [7], as the proof readily indicates.
} 


\section{Note}

A Memorial Service for Pere Menal was given at Universitat Autónoma de Barcelona on October 9, 1991. A memorial booklet contains the moving and beautiful reminiscences of two of his students, and colleagues, Jaume Moncasi and Pere Ara, and an additional commentary by his Ph.D. adviser, and the Director of CRM, Manuel Castellet. I am indebted to Warren Dicks for kindly sending me this commemoration.

I am deeply moved that I was able to be a small part of the life of this inspirational and noble soul, a man of true genius and great learning and vision.

\section{REFERENCES}

1. J. Bjork, Rings satisfying certain chain conditions, J. Reine Angew. Math. 245 (1971), 63-73.

2. P. M. Cohn, Skew field constructions, Cambridge Univ. Press, London, New York, and Melbourne, 1977.

3. J. H. Cozzens, Homological properties of the ring of differential polynomials, Bull. Amer. Math. Soc. 76 (1970), 75-79.

4. C. Faith, Algebra II: ring theory, Springer-Verlag, Berlin, Heidelberg, and New York, 1976.

5. S. M. Ginn, A counter-example to a theorem of Kurshan, J. Algebra 40 (1976), 105-106.

6. S. M. Ginn and P. B. Moss, Finitely embedded modules over Noetherian rings, Bull. Amer. Math. Soc. 81 (1975), 709-710.

7. B. Johns, Annihilator conditions in Noetherian rings, J. Algebra 49 (1977), 222-224.

8. R. P. Kurshan, Rings whose cyclic modules have finitely generated socle, J. Algebra 15 (1970), 376-386.

9. B. L. Osofsky, On twisted polynomial rings, J. Algebra 18 (1971), 597-607.

10. R. Resco, Division rings and V-domains, Proc. Amer. Math. Soc. 99 (1987), 427-431.

Department of Mathematics, Rutgers University, New Brunswick, New Jersey 08903 Current address, Carl Faith: Department of Mathematics, Rutgers, The State University, New Brunswick, New Jersey 08903

Centre de Recerca Matemàtica, Institut d'Estudis Catalans, Bellaterra, Barcelona, SPain

Address all queries for Pere Menal to either the senior author, or to Jaume Moncasi: Department de Matemàtiques, Universitat Autònoma de Barcelona, Bellaterra 08193, Barcelona, Spain 